\title{
ARQUEOLOGÍA DE LA EDAD DEL BRONCE EN ALICANTE: LA HORNA, FOIA DE LA PERERA Y LLOMA REDONA
}

\author{
ANA PUIGCERVER HURTADO \\ Universidad de Alicante
}

\begin{abstract}
Se estudian los registros faunísticos de tres yacimientos de la Edad del Bronce, con el desarrollo de la ganadería doméstica, sobre todo ovicaprinos, y el mantenimiento de la caza.

We have studied the fauna records in three beds from Age of Bronze, together with the development of domestic stockbreeding, above all sheep and goats, and the maintenance of hunting.
\end{abstract}

El estudio zooarqueológico de los tres poblados de la Edad del Bronce situados en las comarcas del sur del País Valenciano, se integra en un proyecto de la Cátedra de Prehistoria de la Universidad de Alicante que intenta abordar la cuestión de las fronteras entre el Bronce Argárico y el Bronce Valenciano.

La Lloma Redona y La Horna, localizados en el Medio Vinalopó, y la Foia de la Perera, en la comarca de la Foia de Castalla, se integran en un período cultural que en otras áreas, como el sureste donde la Cultura Argárica está firmemente implantada, las actividades agrarias tienen un importante desarrollo caracterizado por la generalización de los productos secundarios en la ganadería. Los yacimientos se sitúan en un territorio que Tarradell en 1962 estableció como límite norte de la Cultura Argárica, dado la presencia de poblados como Cabezo Redondo de
Villena con claros elementos culturales característicos de esta cultura.

Los escasos estudios zooarqueológicos en el sur del País Valenciano sobre la Edad del Bronce hacían imprescindible este tipo de trabajos que puedan llegar a elaborar alguna hipótesis sobre los modos de producción ganadera de estos grupos humanos del II milenio a.C. y comenzar a delimitar las afinidades o diferencias con las culturas limítrofes.

\section{METODOLOGÍA}

El proceso de análisis y estudio se inició tras la limpieza y signado de los restos faunísticos. En primer lugar, procedimos a la descripción, determinación de la especie, cálculo del número mínimo de individuos, cálculo de la edad y a la toma de medidas de todos los restos mensurables. 
Todos los restos han sido determinados por nosotros, siempre contando con la ayuda de Manuel Pérez Ripoll ante cualquier duda planteada. Asimismo, utilizamos el material osteológico comparativo para lograr una mejor identificación de las especies, así como para poder esclarecer el difícil problema de la diferenciación entre cabras y ovejas domésticas, cuestión sobre la que también trabajamos con el estudio de J. Boessneck publicado en «Ciencia en Arqueología» (BOESSNECK, 1980).

En cuanto a la determinación de la muerte de los animales domésticos, para el caso del caballo escogimos las pautas establecidas por Jesús Altuna (ALTUNA, 1978). Con el ganado bovino hemos seguido el esquema planteado por Grigson (GRIGSON, 1982), basado en el estudio de la fusión de las epífisis del esqueleto postcraneal y en el estado de desarrollo y desgaste de la dentición. Para calcular la edad de muerte del ganado ovicaprino nos hemos servido del trabajo de Ewbank, Philipson y Whitehouse con Higgs (EWBANK et alii, 1964), en el que se trabaja sobre mandíbulas y maxilares. Para asignar la edad del cerdo doméstico en el momento de su sacrificio hemos utilizado los criterios propuestos por Bull (BULL, 1982), basado en la erupción de los dientes y molares, así como en la fusión del esqueleto postcraneal.

Para calcular el número mínimo de individuos (NMI) reunimos en cada nivel las piezas óseas más frecuentes de cada especie, luego separamos las que eran del lado derecho e izquierdo. El NMI de cada especie animal será siempre el que más se repita del lado derecho o izquierdo. También hemos establecido matices sobre el estado de soldadura de los huesos y, si son mandíbulas, al estado de la dentición. Cuando en un nivel no logramos apoyarnos en ninguno de los criterios descritos, por la poca información ofrecida por esos restos, establecemos un mínimo de un individuo por cada una de las especies de que se tratase.

Hemos medido todos los restos mensurables, a excepción de los huesos quemados y de aquellos otros que correspondían a individuos muy jóvenes, para ello utilizamos la metodología establecida por Angela von den Driesch (von den DRIESCH, 1976).

\section{LOS POBLADOS}

El primer poblado estudiado es la FOIA de la PERERA en Castalla del que se han registrado un total de 178 restos óseos, de los que hemos podido determinar 93, correspondiendo a 17 individuos. La distribución de los restos y del NMI por especies es la que a continuación se detalla:

\begin{tabular}{lrrcr}
\hline & NR & $\%$ & NMI & $\%$ \\
\hline Bos taurus & 2 & 2,1 & 1 & 5,8 \\
Ovicápridos & 83 & 89,2 & 9 & 52,9 \\
Ovis aries & 1 & 1,0 & 1 & 5,8 \\
Sus domesticus & 2 & 2,1 & 2 & 11,5 \\
Canis familiaris & 1 & 1,0 & 1 & 5,8 \\
Oryctolagus cuniculus & 4 & 4,3 & 3 & 17,6 \\
TOTAL & 93 & & 17 & \\
\hline
\end{tabular}

Los ovicápridos son mayoritarios entre las especies determinadas en el poblado. El buey, con dos restos identificados, aparece con el mismo número de restos que el cerdo. Un resto de oveja y otro de perro cierran la lista de especies domésticas identificadas. El conejo es el único animal salvaje presente en el poblado, constituye el $4,3 \%$ del total de restos identificados y, a pesar de la diferencia numérica con los ovicápridos, es la segunda especie más importante en número de restos de la Foia de la Perera.

El material faunístico de la Foia de la Perera se ha caracterizado por su alto grado de fragmentación y se ha localizado en un área muy reducida del poblado. Los cortes 4 y 8 cuentan con aproximadamente el $80 \%$ de los restos faunísticos con los que hemos podido trabajar. Ambos espacios recogen una buena parte de restos constructivos y de habitat, parte del corte 4 se sitúa fuera de una posible cabaña, mientras que el corte 8 ocupa todo el área interior de una vivienda de la Edad del Bronce.

El segundo poblado que integra nuestro trabajo es la LLOMA REDONA, situado en el término municipal de Monforte del Cid, en la comarca del Medio Vinalopó. El poblado ha registrado 77 restos óseos, de los que han podido identificarse 42 , calculando un número mínimo de 21 individuos.

\begin{tabular}{lrrrr}
\hline & NR & $\%$ & NMI & $\%$ \\
\hline Ovicápridos & 33 & 78,5 & 14 & 66,6 \\
Capra hircus & 1 & 2,3 & 1 & 4,7 \\
Sus domesticus & 1 & 2,3 & 1 & 4,7 \\
Oryctolagus cuniculus & 7 & 16,6 & 6 & 23,8 \\
TOTAL & 42 & & 21 & \\
\hline
\end{tabular}

La Lloma Redona se ha caracterizado por la escasez de material, la dispersión de los restos y su mal estado de conservación que han impedido esta- 
blecer conclusiones más substanciales sobre las actividades ganaderas del poblado.

Los ovicápridos, con el 78,5\% de los restos identificados, es la especie más abundante, identificamos un resto de cabra doméstica y otro de cerdo. Como hemos observado en la Foia de la Perera, el conejo es la única especie salvaje presente en el poblado y, por su número de restos, la más importante tras los ovicápridos.

El último poblado que se presenta en este artículo se localiza en el municipio de Aspe, dentro de la comarca del Medio Vinalopó. Durante la excavación de LA HORNA se recogieron un total de 870 restos, de los cuales se determinaron 495, que corresponden a 90 individuos.

\begin{tabular}{lrrrr}
\hline & NR & $\%$ & NMI & $\%$ \\
\hline Equus caballus & 8 & 1,6 & 4 & 4,4 \\
Bos taurus & 53 & 11,5 & 11 & 12,2 \\
Ovicápridos & 342 & 69,0 & 36 & 40,0 \\
Capra hircus & 6 & 1,2 & 6 & 6,6 \\
Ovis aries & 4 & 0,8 & 4 & 4,4 \\
Sus domesticus & 8 & 1,6 & 6 & 6,6 \\
Canis familiaris & 8 & 1,6 & 3 & 3,3 \\
Oryctolagus cuniculus & 48 & 9,6 & 13 & 14,4 \\
Lepus capensis & 5 & 1,0 & 4 & 4,4 \\
Cervus elaphus & 8 & 1,6 & 2 & 2,2 \\
Capra pyrenaica & 1 & 0,2 & 1 & 1,1 \\
TOTAL & 495 & & 90 & \\
\hline
\end{tabular}

El registro faunístico de La Horna destaca por su variedad taxonómica en relación a poblados como la Foia de la Perera o la Lloma Redona.

Las especies domésticas dominan en el poblado en cuanto a los esfuerzos dedicados a su producción, especialmente en lo que se refiere a los ovicápridos que, con el $69,0 \%$ de los restos determinados, indica un predominio de la ganadería ovicaprina. El segundo animal más importante en número de restos es el buey. Le siguen a distancia el cerdo, el caballo y el perro.

La presencia de animales salvajes demuestra la permanencia de la actividad cazadora durante la Edad del Bronce, especialmente dedicada a la captura de conejos, aunque especies como el ciervo, la cabra salvaje y la liebre son cazados ocasionalmente, no así el ciervo, pues su número de restos respecto a las otras dos especies salvajes es relativamente importante.
El mayor número de restos óseos se han exhumado de los cortes 3 y E-1.2 de La Horna. Pero, en general, el poblado muestra una alta dispersión de materiales faunísticos, en relación directa a la extensa área excavada. Este aspecto ha concedido más peso al resultado del número mínimo de individuos, como ocurre en el caso de Lloma Redona.

\section{LAS ESPECIES}

\section{EL CABALLO, Equus caballus.}

\begin{tabular}{lrccr}
\hline & NR & $\%$ & NMI & $\%$ \\
\hline La Horna & 8 & 1,6 & 4 & 4,4
\end{tabular}

Sólo han aparecido restos de caballo en La Horna, estando ausentes de la Foia de la Perera y Lloma Redona.

La mayoría de los huesos identificados en La Horna corresponden a los tarsos y metatarsos de los animales. Asimismo, se ha identificado el fragmento de la epífisis distal de un fémur, un incisivo y el fragmento de un molar.

Todos los autores coinciden en que el caballo es un animal doméstico durante la Edad del Bronce (GIL-MASCAREL, 1992). La presencia de este animal en La Horna no nos ha permitido precisar su carácter doméstico, ni precisar el papel del caballo en el seno del grupo humano, pues los restos analizados no han dado a conocer sus modos de explotación y sacrificio.

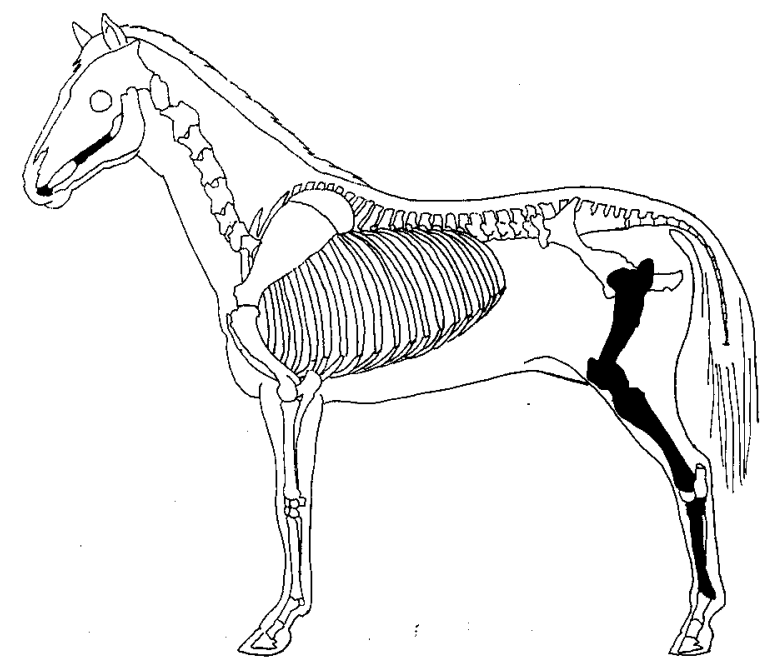



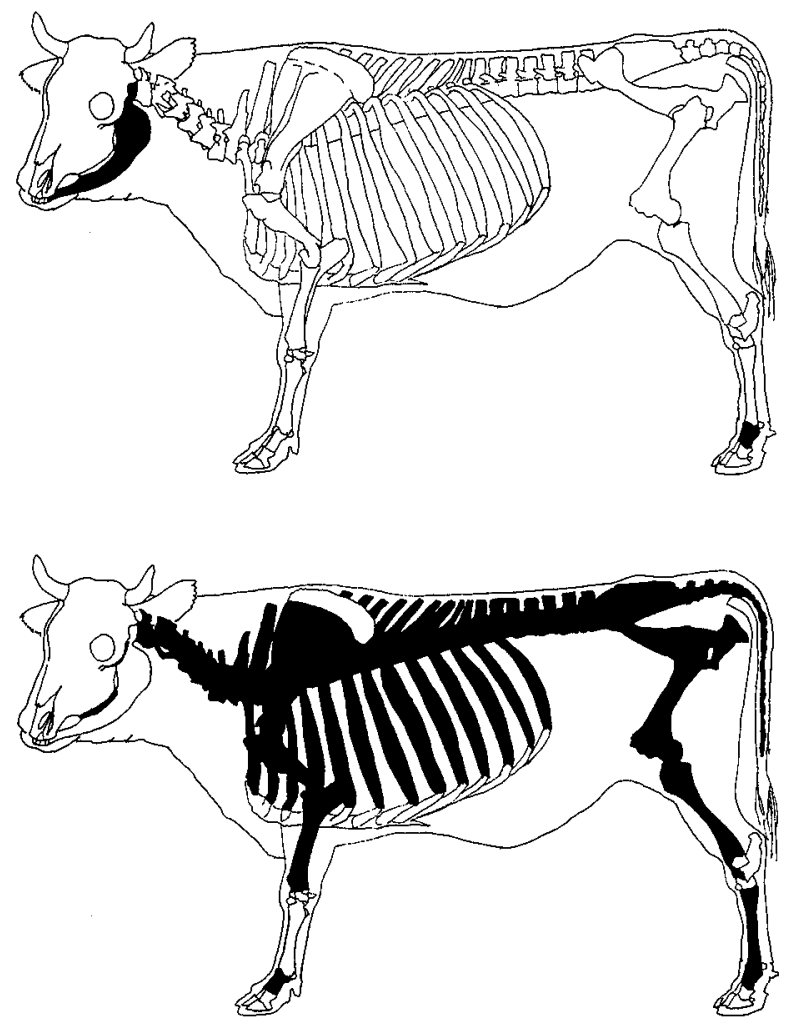

EL BOVINO, Bos taurus.

\begin{tabular}{lrrrr}
\hline & NR & $\%$ & NMI & $\%$ \\
\hline La Foia de la Perera & 22, & 1 & 1 & 5,8 \\
La Horna & 53 & 11,5 & 11 & 12,2 \\
\hline
\end{tabular}

El bovino ocupa un lugar importante entre los animales domésticos identificados. Tras los ovicápridos son los mejor representados en los yacimientos estudiados.

Los dos huesos de bovino registrados en la Foia de la Perera corresponden a un fragmento de mandíbula y a un fragmento longitudinal de falange I.

En La Horna los restos óseos más frecuentes son los de las extremidades traseras, seguidas por las costillas, los huesos de las extremidades anteriores y las vértebras.

El poblado de La Horna ha ofrecido datos sobre la edad en el momento de su muerte de tres individuos. Uno de ellos fue sacrificado cuando había alcanzado la edad subadulta, probablemente el animal fue destinado al consumo. Los otros dos individuos murieron en edad adulta, aunque no eran viejos. De estos datos no podemos establecer un único comportamiento, pero sí podemos plantear la doble utilización que se puede dar a la crianza del ganado bovino: además de servir de alimento, puede ser aprovechado en la producción de leche y pieles o como animal de tiro en las tareas agrícolas.

\section{LOS OVICÁPRIDOS}

\begin{tabular}{lllll}
\hline & NR & $\%$ & NMI & $\%$ \\
\hline & & & & \\
La Foia de la Perera & 93 & 89,2 & 9 & 52,9 \\
La Lloma Redona & 33 & 78,5 & 14 & 66,6 \\
La Horna & 342 & 69,0 & 36 & 40,0 \\
\hline
\end{tabular}

Los resultados obtenidos en los yacimientos estudiados muestran la importancia que adquirió la ganadería ovicaprina durante el II milenio.

En la Foia de la Perera y la Lloma Redona, los huesos más numerosos son las costillas, seguidas de las extremidades traseras y, a continuación, las delanteras. En La Horna las extremidades delanteras es la parte del esqueleto mejor representado en el conjunto. En la Foia de la Perera le siguen los dientes sueltos y, por último, los restos de mandíbula. En la Lloma Redona se ha identificado, además, dos fragmentos de mandíbula y los restos de un diente.

En La Horna la variabilidad anatómica es bastante mayor; así, los dientes sueltos, los restos de cuernos y mandíbulas, las vértebras, la escápula, los fragmentos de pelvis y los de cráneo cierran la lista de elementos identificados, en orden de importancia.

En la Lloma Redona no hemos podido extraer conclusiones sobre los modelos de explotación de estos pequeños rumiantes a través del patrón de sacrificio, al no contar con elementos sobre los que poder valorar.

En la Foia de la Perera hemos podido calcular la edad de muerte en siete de los nueve individuos identificados; de ellos, cuatro fueron sacrificados jóvenes, dos murieron en edad subadulta y, el séptimo individuo fue el único que consiguió alcanzar la edad madura.

En La Horna la edad de muerte se produjo mayoritariamente cuando el animal no se ha desarrollado totalmente; dos individuos se sacrificaron jóvenes, cinco no alcanzaron la edad madura y sólo dos murieron viejos. 

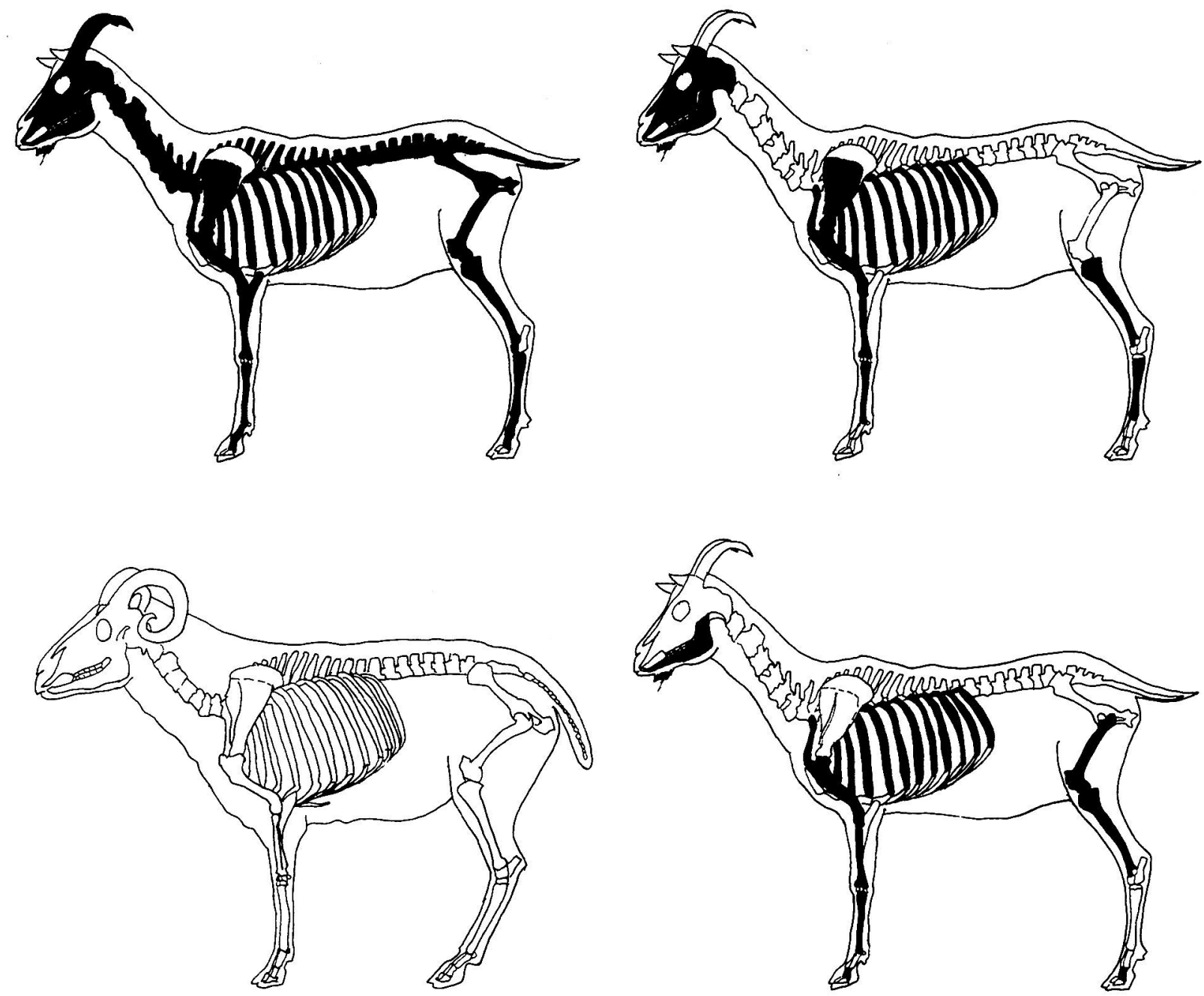

Los datos obtenidos del análisis del patrón de sacrificio en la Foia de la Perera y en La Horna muestran que la cría de ovejas y cabras estaba orientada fundamentalmente al consumo.

\section{LA CABRA, Capra hircus.}

\begin{tabular}{lllll}
\hline & NR & $\%$ & NMI & $\%$ \\
\hline Lloma Redona & 1 & 2,3 & 1 & 4,7 \\
La Horna & 6 & 1,2 & 6 & 6,6 \\
\hline
\end{tabular}

Pocos han sido los restos de ovicápridos diferenciados. A excepción de la Foia de la Perera, donde sólo hemos podido diferenciar restos de oveja, todos los demás poblados estudiados presentan un mayor porcentaje de cabras que de ovejas entre sus restos.

En la Lloma Redona se ha identificado un fragmento de metatarso. En La Horna la mayor parte de los restos óseos identificados son metatarsos, también se pudo distinguir los fragmentos de un cuerno y de una falange I.

\section{LA OVEJA, Ovis aries.}

\begin{tabular}{lllll}
\hline & NR & $\%$ & NMI & $\%$ \\
\hline Foia de la Perera & 1 & 1,0 & 1 & 5,8 \\
La Horna & 4 & 0,8 & 4 & 4,4 \\
\hline
\end{tabular}

No hemos podido distinguir ningún resto de oveja en la Lloma Redona. En la Foia de la Perera el único resto identificado corresponde a una falange II entera.

En La Horna la oveja fue identificada a través de los restos de dos falanges II, un metatarso y un cuerno. 


\section{EL CERDO, Sus domesticus.}

\begin{tabular}{lcccr}
\hline & NR & $\%$ & NMI & $\%$ \\
\hline Foia de la Perera & 2 & 2,1 & 2 & 11,5 \\
Lloma Redona & 1 & 2,3 & 1 & 4,7 \\
La Horna & 8 & 1,6 & 6 & 6,6 \\
\hline
\end{tabular}

El ganado porcino es otra de las especies domésticas que aparece en todos los yacimientos estudiados.

En la Foia de la Perera identificamos el fragmento de una mandíbula y los restos de un diente. En la Lloma Redona el fragmento de una ulna es el único resto determinado de esta especie.

La mayor parte de los huesos de cerdo identificados en La Horna corresponden a restos de dentición suelta; en segundo lugar, dos huesos craneales, seguidos de un fragmento de diáfisis de radio, el de una ulna y la diáfisis de un fémur.

El único individuo con edad de muerte determinada pertenece a La Horna y éste se sacrificó cuando todavía era muy joven. Este dato, aunque muy sesgado, puede confirmar la dedicación del ganado porcino al consumo; el cerdo, aunque no tiene una importancia numérica tan relevante como las anteriores especies comentadas, puede cumplir un papel fundamental en la dieta y economía de estos grupos prehistóricos, al reforzar la dieta tradicional de pequeños rumiantes y tratarse de una ganadería perfectamente próspera en un medio agrícola.

\section{EL PERRO, Canis familiaris.}

\begin{tabular}{lcccc}
\hline & NR & $\%$ & NMI & $\%$ \\
\hline Foia de la Perera & 1 & 1,0 & 1 & 5,8 \\
La Horna & 8 & 1,6 & 3 & 3,3 \\
\hline
\end{tabular}

Un diente suelto es el único testimonio de perro en la Foia de la Perera. Los huesos de los tres individuos de La Horna se distribuyen entre cuatro restos de extremidades anteriores, dos dientes sueltos y dos fragmentos de extremidades posteriores.

El perro parece ser el único animal doméstico no consumido en los poblados estudiados. Su presencia debe estar relacionada con el desarrollo de la ganadería en la Edad del Bronce, en la que cumple un papel de apoyo en las tareas de pastoreo.
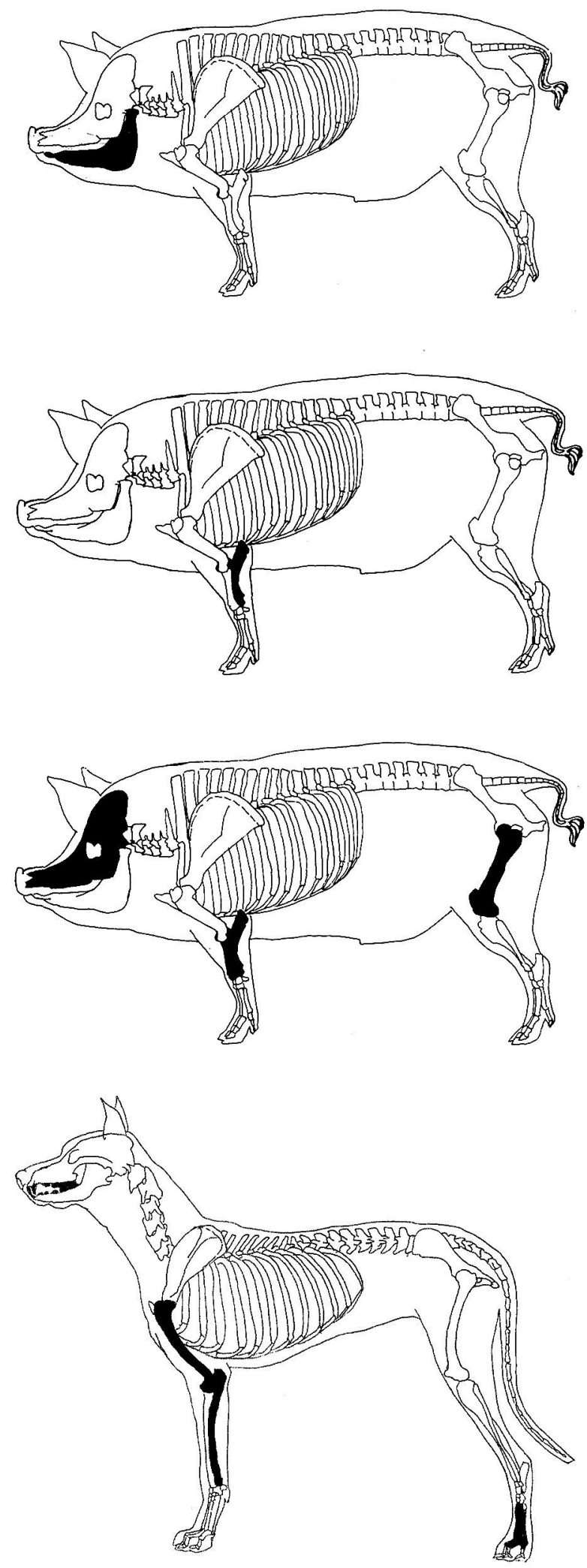
EL CONEJO, Oryctolagus cuniculus.

\begin{tabular}{lrrrr}
\hline & NR & $\%$ & NMI & $\%$ \\
\hline Foia de la Perera & 4 & 4,3 & 3 & 17,6 \\
Lloma Redona & 7 & 16,6 & 5 & 23,8 \\
La Horna & 48 & 9,6 & 13 & 14,4 \\
\hline
\end{tabular}

El conejo es el único animal salvaje presente en todos los yacimientos de la Edad del Bronce estudiados. Se trata de un animal con un habitat muy amplio, desde el bosque a zonas de costa o terrenos cultivados.

Los cuatro restos de conejo de la Foia de la Perera se distribuyen entre dos fragmentos de pelvis, una ulna y el fragmento de un metapodio.

En la Lloma Redona, a excepción de una pelvis, la totalidad de los huesos pertenecen a las extremidades posteriores.

En La Horna más de la mitad de los restos pertenecen a las extremidades posteriores, a cierta distancia se sitúan las extremidades anteriores; por último, se identificaron cuatro mandíbulas y otras tantas pelvis.

\section{LA LIEBRE, Lepus capensis.}

\begin{tabular}{lrlcr}
\hline & NR & $\%$ & NMI & $\%$ \\
\hline La Horna & 5 & 1,0 & 4 & 4,4 \\
\hline
\end{tabular}

La parte corporal que con más frecuencia ha aparecido en La Horna han sido las extremidades posteriores. También identificamos los restos de una vértebra.

\section{EL CIERVO, Cervus elaphus.}

\begin{tabular}{lcclc}
\hline & NR & $\%$ & NMI & $\%$ \\
\hline La Horna & 8 & 1,6 & 2 & 2,2 \\
\hline
\end{tabular}

Tras el conejo, el ciervo es el animal que con más frecuencia aparece en los poblados de la Edad del Bronce. Su habitat es el de los bosques caducifolios y también se integran perfectamente en los bosques mixtos y abiertos.

La distribución anatómica de los restos óseos del ciervo en La Horna está muy equilibrada entre las extremidades anteriores y posteriores, así como entre los dientes sueltos.

Los restos de dentición han proporcionado la edad de muerte de dos individuos. Ambos fueron cazados cuando habían alcanzado los tres años, en
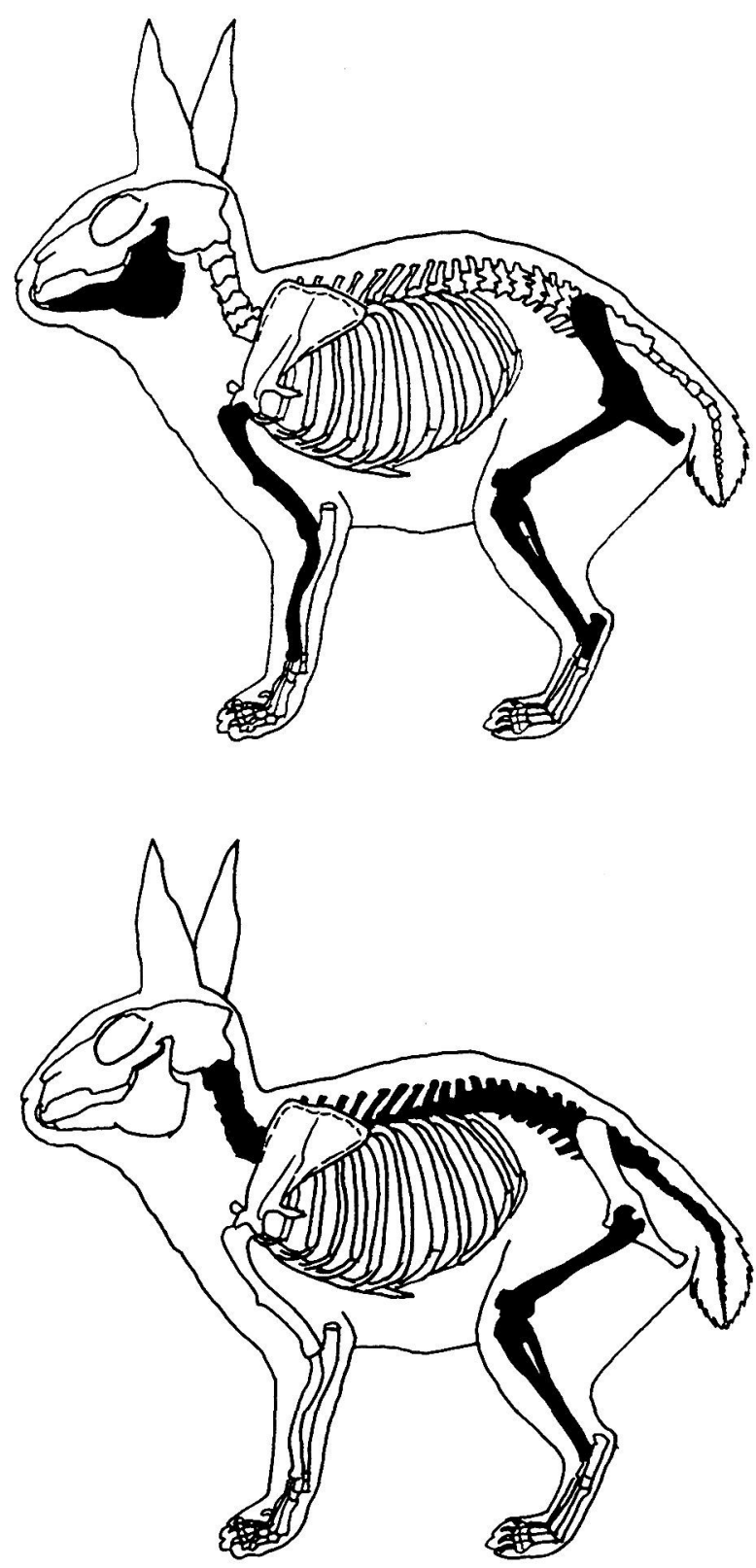

este estadio se encuentran en unas condiciones aptas para el consumo, ya que el animal está maduro y completamente desarrollado, pero sus carnes no están endurecidas.

\section{LA CABRA SALVAJE, Capra pyrenaica.}

\begin{tabular}{rrrrr}
\hline & NR & $\%$ & NMI & $\%$ \\
\hline La Horna & 1 & 0,2 & 1 & 1,1 \\
\hline
\end{tabular}



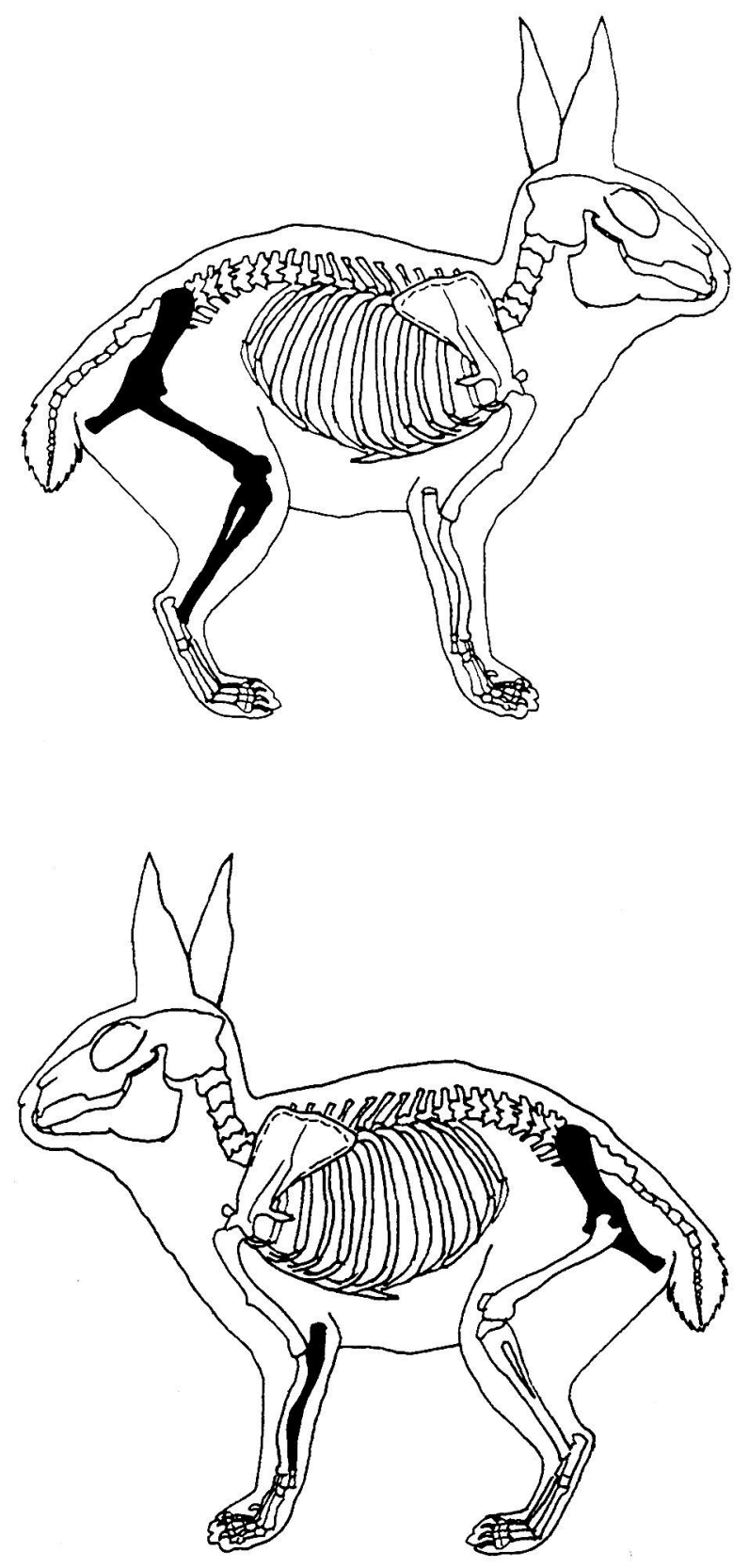

La presencia de la cabra salvaje es muy marginal. Su caza sería muy esporádica, dado que habría que ir a buscarlas a zonas de alta montaña y riscos de gran altura, por lo que se tendrían que producir desplazamientos relativamente lejanos de los poblados.

El resto óseo de la cabra salvaje clasificado en La Horna corresponde a una falange I.

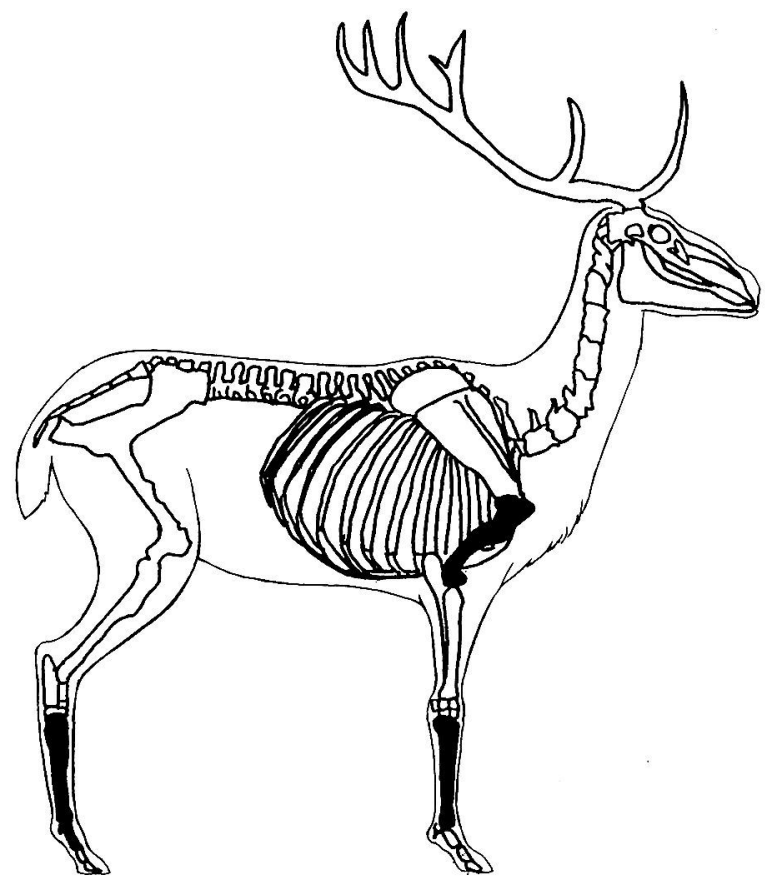

\section{CONCLUSIONES}

Uno de los problemas con los que nos hemos encontrado al enfrentarnos al estudio zooarqueológico de estos tres yacimientos de la Edad del Bronce ha sido el deficiente estado de conservación de los materiales, lo que ha coartado la posibilidad de profundizar sobre las características de cada una de las especies, las relaciones entre ellas y los modos de explotación; de manera particular, cuando hemos querido realizar el estudio del sexo, con el propósito de definir mejor el patrón de sacrificio que se ejercía sobre la ganadería doméstica.

El desarrollo de la ganadería doméstica durante la segunda mitad del II milenio aparece bien atestiguada en todos los poblados. Esta se basa en la cría de ovicaprinos para el consumo. Los bóvidos pudieron ser utilizados para suministro de carne, pero los dos individuos sacrificados en edad adulta en La Horna, tal vez fueron destinados previamente a la producción de leche u otro tipo de actividad económica. El tercer animal que caracteriza la economía ganadera es el cerdo, con una dedicación exclusiva al consumo, en un intento de hacer más variada la dieta cárnica. Para terminar con los animales domésticos, nos resulta difícil explicar el papel del caballo en estos poblados de la Edad del Bronce, al no contar con suficientes datos que permitan determinar cómo son explotados. 
Paralelamente a la cría de animales domésticos, estos poblados de la segunda mitad del II milenio a.C. muestran el mantenimiento de la caza. Esta actividad económica, fundamental en épocas precedentes por ser la única fuente de suministro de carne, pasa durante la Edad del Bronce a un segundo plano, como resultado del desarrollo de la ganadería. La caza aumenta la riqueza de la dieta cárnica; del mismo modo, su perduración en el tiempo, puede responder a la necesidad de conservar una tradición cultural o a la de defender los cultivos de los animales depredadores.

\section{BIBLIOGRAFÍA}

ALTUNA, J., 1980: «Historia de la domesticación animal en el País Vasco desde sus orígenes hasta la Romanización». Munibe, 32. San Sebastián.

ARTEAGA, O. y SCHUBART, H., 1986: «Fundamentos para el estudio socio-económico y cultural del área del Argar». Homenaje a L. Siret; pp. 289-307. Sevilla.

BENITO, M., 1989: «Estudio de un pequeño conjunto óseo del poblado de El Pic dels Corbs (Sagunto)». En BARRACHINA, A., 1989: «Breve avance sobre el estudio de El Pic dels Corbs». Homenaje a Chabret, 1888-1988. Valencia.

BOESSNECK, J., 1969: «Restos óseos del cerro de la Virgen de Orce y del Cerro del Real, Galera (Granada)». NAH, X, XI y XII (pp. 172 y ss.). Madrid.

-1980: «Diferencias osteológicas entre las ovejas (Ovis aries Linné) y las cabras (Capra hircus Linné)» En

BROTHWELL, D.; HIGGS, E. y CLARK, G. (compiladores) 1980: Ciencia en Arqueología, Fondo de Cultura Económica.

BLLL, G. y PAYNE, S., 1982: «Tooth eruption and epiphysial fusion in pigs and wild board». En WILSON, GRIGSON and PAYNE: «Ageing and sexing animals bones from archeological sites», pp. 55-73. BAR, 109. Oxford, England.

BURTON, M., 1985: Guía de los mamíferos de España y de Europa. Ediciones Omega. Barcelona.

DAVIS, S.J.M., 1989: La arqueología de los animales, Ediciones Bellaterra, Barcelona.

DRIESCH, A. y BOESSNECK, J., 1969: «Die fauna des Cabezo Redondo bei Villena (provincia de Alicante)». Studien über frïher Tierknochenfunden von der Iberischen Halbinsel, pp. 4389. München.

ENGUIX, R., 1975: «Notas sobre la economía del Bronce Valenciano». Aniversario de la fundación del Laboratorio de Arqueología. 1924-74. PLAV, 11, pp. 141-157.

EWBANK, J.; PHILLIPSON, P. and WHITEHOUSE, R. whith HIGGS, E., 1964: «Sheep in the Iron Age: a method of study». Proc. Prehist. Soc. (N.S.) 30, 423-b.

FORTEA et alii, 1988: «Epipaleolítico y Neolitización en la zona oriental de la Península Ibérica». Montpellier.

GIL-MASCARELL, M., 1981: «El Bronce tardío final en el País Valenciano». Monografias del Laboratorio de Arqueología de Valencia, 1, pág. 133. Valencia.
- 1992: «La agricultura y la ganadería como vectores económicos del desarrollo del Bronce Valenciano». Saguntum, 25, pp. 49-69. Valencia.

GIL-MASCARELL, M. y ENGUIX, R., 1986: «La cultura del Bronce valenciano: estado actual de la investigación» Homenaje a L. Siret; pp. 418-424. Sevilla.

GRIGSON, C., 1982: «Sex and age determination of some bones and teeth of domestic cattle: a review of the literature». En WILSON, GRIGSON and PAYNE. BAR, 109. Oxford, England.

HERNÁNDEZ, M.S., 1985: «La Edad del Bronce en el País Valenciano». Arqueologia en el País Valenciano. Panorama y Perspectivas. Anejo rev. Lucentum, Alicante.

- 1986: «La Horna (Aspe)». Arqueología en Alicante (1976-86). Alicante.

- 1986: «La Cultura de El Argar en Alicante. Relaciones temporales y espaciales con el mundo del Bronce valenciano». Homenaje a $L$. Siret. Sevilla.

JUAN, J. y MARTÍNEZ, R., 1988: «Fuente Flores (Requena, Valencia). Nuevos datos sobre la economía del Neo-Eneolítico valenciano». $A P L$, XVIII. Valencia.

LULL, V., 1984: «La cultura del Argar (un modelo para el estudio de las formaciones económico-sociales)». Ed. Akal. Madrid.

MARTI, B., 1983: «El nacimiento de la agricultura en el País Valenciano». Universidad laboral, secretariado de publicaciones. Universidad de Valencia, Valencia.

MARTÍNEZ, R., 1988: «El estudio de la fauna de dos yacimientos ibéricos Villares y el Castellet de Bernabé». Saguntum, 21, Valencia.

NAVARRO, J.F., 1983: «La explotación del territorio en la Península Ibérica durante el Bronce Pleno. Aproximación a su estudio». Rev. Tabona, n. 4. Universidad de La Laguna, Tenerife.

PÉREZ, M., 1981: «Análisis faunístico de los restos óseos procedentes del poblado de la Edad del Bronce de Les Planetes». CPAC, 5, pp. 238-21. Castellón.

- 1990: «La ganadería y la caza en la Ereta del Pedregal (Navarrés, Valencia)» $A P L, \mathrm{XX}$. Valencia.

- 1991: «Estudio zooarqueológico» En: La cueva del Lagrimal (Villena, Alicante) de José María Soler. C.A.P.A., Alicante.

- 1992: Marcas de carnicería, marcas intencionadas y mordeduras de carnivoros en huesos prehistóricos del Mediterráneo español Instituto de Cultura «Juan GilAlbert». Diputación Provincial de Alicante. Alicante.

SOLER, J.M., 1984: «La Edad del Bronce en la comarca de Villena». Homenaje a L. Siret. Sevilla.

- 1987: «Excavaciones arqueológicas en el Cabezo Redondo (Villena, Alicante)». Ayuntamiento de Villena. Instituto de Cultura «Juan Gil-Albert». Alicante.

SORIANO, R., 1984: «La Cultura del Argar en la Vega Baja del Segura». Saguntum, 18. Valencia.

- 1985: «Contribución al estudio del Bronce tardío y final en la Vega Baja del Segura». Saguntum, 19. Valencia.

TARRADEL, M., 1962: El País Valenciano del Neolítico a la Iberización. Ensayo de sintesis. Facultad de Filosofía y Letras. Cuaderno II. Universidad de Valencia. Valencia.

- 1965: «El problema de las diversas áreas culturales en la Península Ibérica durante la Edad del Bronce» Miscelánea Breuil, II, pp. 421. Barcelona.

- 1969: «La cultura del Bronce Valenciano. Nuevo ensayo de aproximación». PLAV, 6, pp. 7-30. Valencia

UCKO, P. y DIMBLEDY, G.W., 1969: The domestication and explotation of plants and animal. London. 